\title{
Spectral Vacancies Prediction Method for Cognitive Radio Applications
}

\author{
A. Canavitsas, L. da Silva Mello, M. Grivet \\ Pontifical Catholic University of Rio de Janeiro, Rua Marques de S. Vicente, 225 - 22.451-900 - Rio de Janeiro, \\ Brazil-smello@cetuc.puc-rio.br
}

\begin{abstract}
Cognitive radio technology is in fast development and is considered a possible solution to improve the efficiency of radio spectrum use. Many studies have been recently carried out in order to improve spectrum-sharing techniques between primary and secondary users. This paper investigates one of the basic decision problems faced by a cognitive radio: given a time window of a specific size, a secondary user (SU) should decide if it will use it or not, minimizing the chances of collision with a primary user (PU). For this purpose, an algorithm is proposed that does not require previous information about PU occupancy behavior. The proposed algorithm shows very good performance when compared to three other methods recently proposed to tackle with this problem.
\end{abstract}

Index Terms - Spectral vacancies, spectrum sharing, cognitive radio.

\section{INTRODUCTION}

It is well known that frequency bands solely allocated to primary services (licensed spectrum holders) are generally underused and limit the development of new telecommunications services. As discussed by Thomas [1], there is still much conservatism in the simultaneous use of spectrum bands by primary and secondary users in terms of licensing and protection against interference.

Cognitive radio has been proposed as a technology for dealing with spectrum-sharing problems and improving the efficiency spectrum use. The prediction of the occurrence of white spaces, defined as time intervals during which a frequency channel will go unoccupied by the primary user in a certain geographic region, is an existing challenge to be overcome in order to enable an optimized operation of these systems. The sensing of the channel and the decision to use it on a single time-slot basis generates severe latency due to the processing and coordination involved and can reduce the transfer rate of desired information. Therefore, this decision should be taken for continuous blocks of timeslots.

The prediction method proposed in this paper deals with the most basic decision problem faced by cognitive radios: given a time window of a specific size, the secondary user (SU) should decide if it will use it or not, keeping to a minimum the probability of collision with the primary user (PU). The information that the SU has access to is the past-observed behavior of the PU.

Section II of the paper reviews some methods, recently developed by other authors, for solving this problem. Section III formally presents the problem to be solved as well as the proposed solution and the underlying algorithm. Section IV describes the measurements campaign [2] that provided 
spectrum occupancy data to test the proposed algorithm. Section V shows the performance comparison of the proposed method with other methods found in the technical literature. Finally, Section VI presents the conclusions of the work.

\section{RELATED WORKS}

Soleimani et al [3] developed a dynamic handoff procedure based on decreasing handoff rate as well as increasing overall secondary users' throughput. Using a Hidden Markov Model (HMM), the secondary user predicts the next primary user's arrival based on past observation and a model which is learned from past data. The idle probability of target channels is used to improve the accuracy of the scheme. Simulation results show that, considering handoff rate and packet delivery ratio, the proposed scheme is efficient for light loaded traffic.

Game Theory is also a fertile ground to deal with frequency spectrum inefficiencies [4] but as far as the authors could observe, it is more concerned with the situation of spectral sharing by several cognitive users or networks. Furthermore, it does require the definition of utility functions that could be difficult de assess or define.

On these lines, Jing et al [5] investigated cooperative spectrum prediction and local prediction. They proposed a local spectrum prediction method in which each secondary user senses the current channel state and uses a Hidden Markov Model (HMM) to predict the future channel states. They also proposed a cooperative spectrum prediction method based on a coalitional game-based approach, in which the secondary users are considered as players who attempt to form coalitions and cooperatively predict the future channel status. The results indicate that the prediction accuracy can be improved through cooperation.

The recent work of Akhtar el al [6] presents a novel spectrum decision scheme, which according to the authors, remains an unexplored area in cognitive radio research. The proposed decision making scheme is based on the fusion of three key channel parameters i.e. channel idle time, channel occupancy status and channel performance, thereby facilitating the secondary user to occupy the targeted channel without impairing the licensed user's communication while maintaining its own QoS requirements. Decision structural function is used as an evaluation measure to check the robustness of the proposed scheme.

Barnes and Maharaj [7] proposed a method to improve the performance of cognitive radios increasing secondary users flow and reducing data degradation of information sent by the primary user. A channel switching simulator was developed to evaluate the performance of the primary users, wherein the Hidden Markov chain is implemented to model and predict the behavior of primary user. According to then, the performance of the cognitive system can be enhanced if the UP behavior is adequately modeled. Thus, significant gains in system performance can be obtained using a prediction model, particularly in heaviest traffic conditions, when there is an increase of secondary user activity. 
Zao et al [8] combine the sensing and prediction to enhance the spectrum utilization and reduce the interference on the primary user. In addition, they propose an effective solution using the dual optimal theory.

Gulnur et al [9] proposed methods that are based on the correlation and linear regression analysis of the previous decisions, to further forecast the future spectrum status. Since these prediction mechanisms solely depend on individual sensing histories of secondary users, they are suitable for implementation in cognitive radio ad hoc networks.

Guknur and Oktug [10] also propose a method for predicting spectrum holes by defining service classes among CR users and accomplish spectrum sharing mechanism accordingly.

\section{PROPOSED ALGORITHM}

\section{A. Problem statement}

Consider the situation in which a secondary user (SU) wishes to opportunistically use a channel assigned to a primary user (PU). For this purpose, the SU probes the channel, checking at each time slot if it is occupied by PU (busy) or not (idle) in order to capture de PU's occupancy statistical behavior. Considering $t$ as the present moment, SU captures the channel status (busy or idle) at time slots $\mathrm{t}, \mathrm{t}-1, \ldots ., \mathrm{t}-\mathrm{N}$ (observation window) and wishes to decide how to behave (in terms of occupation or not) at time slots $\mathrm{t}+1, \mathrm{t}+2, \ldots, \mathrm{t}+\mathrm{M}$ (prediction window), as indicated in Figure 1. Please notice that no further information is assumed to be known except the observed channel status data during the observation window.

It is important to realize that this work is based on the assumption that the window sizes $\mathrm{M}$ and $\mathrm{N}$ are somehow established and the goal here is to do the best under these circumstances. It is obvious that parameter $\mathrm{M}$ and $\mathrm{N}$ have an impact on the collision statistics, but this is not treated here although it is conceived that some form of optimization can be proposed in order to seek for good values for $\mathrm{M}$ an $\mathrm{N}$ as far the successful occupancy of the prediction window is concerned.

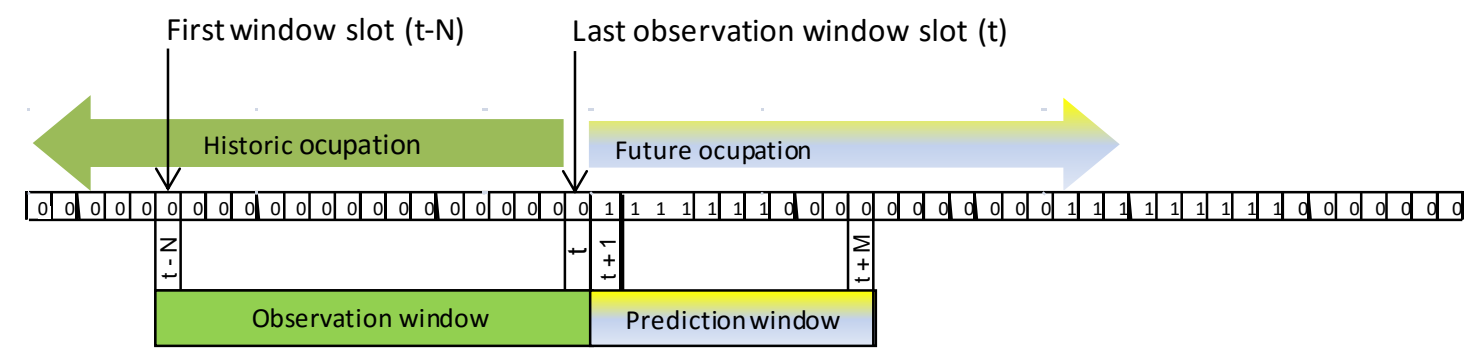

Fig. 1. Historic and future occupation, observation and prediction windows

We initially assume that $\mathrm{M}$ and $\mathrm{N}$ are small when compared with the average times of activity and idle periods exhibited by the PU. This is convenient because one can then assume that it is unlikely that observation and prediction windows do cover more than one pair of busy and idle cycles. Hence, 
denoting by $\mathrm{X}_{\mathrm{k}}$ the channel state due to PU activity ( $0=\mathrm{idle}$; $1=$ busy) and in the absence of occupancy observation errors, there are two possible cases for the observation window:

- Case 1: $\mathrm{X}_{\mathrm{t}-\mathrm{k}}=0$ for all $\mathrm{k}$ in $\{0,1, \ldots \mathrm{L}-1\}$ and $\mathrm{X}_{\mathrm{t}-\mathrm{k}}=1$ for all $\mathrm{k}$ in $\{\mathrm{L}, \mathrm{L}+1, \ldots \mathrm{N}-1\}$

- Case 2: $\mathrm{X}_{\mathrm{t}-\mathrm{k}}=1$ for all $\mathrm{k}$ in $\{0,1, \ldots \mathrm{L}-1\}$ and $\mathrm{X}_{\mathrm{t}-\mathrm{k}}=0$ for all $\mathrm{k}$ in $\{\mathrm{L}, \mathrm{L}+1, \ldots \mathrm{N}-1\}$

Please notice that the cases are direct consequences of the assumption made in the last paragraph.

Nevertheless, in real situations there are observation errors. We assume that each element of the observed sequence $\left\{\mathrm{X}_{\mathrm{t}-\mathrm{k}}, \mathrm{k}=\{0,1, \ldots \mathrm{M}-1\}\right\}$ can be independently corrupted by noise in such a way that state inversion occurs with a fixed probability smaller than $1 / 2$.

These considerations allows us to conclude that the elements of the above sequence can be treated as independent, Bernoulli-distributed random variables where $\left\{X_{t-k}, k=\{0,1, \ldots L-1\}\right\}$ are Bernoulli(p) while $\left\{\mathrm{X}_{\mathrm{t}-\mathrm{k}}, \mathrm{k}=\{\mathrm{L}, \mathrm{L}+1, \ldots \mathrm{N}-1\}\right\}$ are Bernoulli(1-p).

Case 1 corresponds to the situation where $p<1 / 2$ and Case 2 is associated with $p>1 / 2$. So, the problem to solve is this: taking into account the observation window has produced the values $\left\{X_{t}, X_{t-1}\right.$, $\ldots, \mathrm{X}_{\mathrm{t}-\mathrm{N}-\mathrm{l})}$ \} for the channel status, we want to properly estimate the values of $\mathrm{p}$ and $\mathrm{L}$ that will be used for the prediction scheme. The decision of occupying the prediction window will be based upon these two quantities.

\section{B. Proposed solution}

In a nutshell, we follow here the classical statistical methodology [11] where the main idea is to access the likelihood of the observed sequence $\left\{X_{t}, X_{t-1}, \ldots X_{t-(N-1)}\right\}$ as a function of $L$ and $p$ and then finding values for these parameters that lead to its maximization. By doing this, we are pursuing the best estimation line of work when no extra information is given about the data.

Since the observation errors are statistically independent, which is a quite fair assumption, the likelihood of the observed sequence $\underline{X}_{t}^{N}=\left(X_{t}, X_{t 1}, \ldots, X_{t(N 1)}\right)$ is given by:

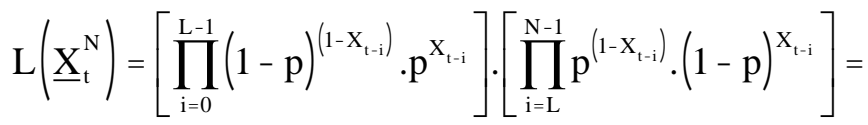

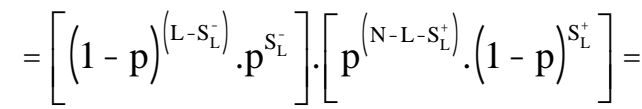

$$
\begin{aligned}
& =\mathrm{p}^{\mathrm{N}\left(\mathrm{L} \mathrm{S}_{\mathrm{L}}+\mathrm{S}_{\mathrm{L}}^{+}\right)} \cdot\left(\begin{array}{ll}
1 & \mathrm{p}
\end{array}\right)^{\left(\mathrm{L} \mathrm{S}_{\mathrm{L}}+\mathrm{S}_{\mathrm{L}}^{+}\right)}=\mathrm{p}^{\mathrm{N}} \quad(\mathrm{L}) \cdot\left(\begin{array}{ll}
1 & \mathrm{p}
\end{array}\right)^{(\mathrm{L})}
\end{aligned}
$$

where:

$$
\begin{aligned}
& \mathrm{S}_{\mathrm{L}}={ }_{\mathrm{i}=0}^{\mathrm{L} 1} \mathrm{X}_{\mathrm{t}} \mathrm{i}
\end{aligned}
$$

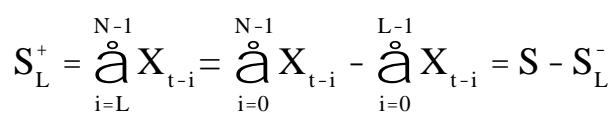

$$
\begin{aligned}
& (\mathrm{L})=\mathrm{L} \quad \mathrm{S}_{\mathrm{L}}+\mathrm{S}_{\mathrm{L}}^{+}=\mathrm{L}+\mathrm{S} \quad 2 . \mathrm{S}_{\mathrm{L}}
\end{aligned}
$$

It is relatively simple to note that: 
- if $(\mathrm{L})=\mathrm{N}$ then $\mathrm{L}\left(\underline{\mathrm{X}}_{\mathrm{t}}^{\mathrm{N}}\right)$ is maximum when $\mathrm{p}=0$

- if $(\mathrm{L})=0$ then $\mathrm{L}\left(\underline{\mathrm{X}}_{\mathrm{t}}^{\mathrm{N}}\right)$ is maximum when $\mathrm{p}=1$

- if $(\mathrm{L}) \notin\{0, \mathrm{~N}\}$ then $\mathrm{L}\left(\underline{\mathrm{X}}_{\mathrm{t}}^{\mathrm{N}}\right)$ is maximum when $\mathrm{p}=1 \frac{(\mathrm{L})}{\mathrm{N}}$

From the above we can conclude that in all cases, $L\left(\underline{X}_{t}^{N}\right)$ is maximized when $p=1 \frac{(L)}{N}$. To avoid possible numeric underflows during its computation, the log likelihood function is used. Its optimal value is then given by:

$$
\mathrm{L}_{\mathrm{opt}}\left(\underline{\mathrm{X}}_{\mathrm{t}}^{\mathrm{N}}\right)=\left(\begin{array}{ll}
\mathrm{N} & (\mathrm{L})
\end{array}\right) \cdot \log \left(1 \frac{(\mathrm{L})}{\mathrm{N}}\right)+(\mathrm{L}) \cdot \log \left(\frac{(\mathrm{L})}{\mathrm{N}}\right)
$$

whose maximum in $\mathrm{L}$ can be found by brute force with low computational cost.

Unfortunately, when the estimated L is small, the sensitivity of the pestimator is often high because small bursts of 0's or 1's can be solely caused by observation errors. To mitigate this undesirable effect, when estimated $\mathrm{L}$ is smaller than a threshold $\mathrm{L}_{\text {bound, }}$, which should be found by experimentation, we decide to estimate $\mathrm{p}$ by means of the empirical error frequency in the whole sequence.

Although rare, it is possible that more than one PU cycle of activity/idleness can occur inside the observation window. Again, this effect can be mitigated by re-applying the (L, p) estimation in the observation sub-window $\left(X_{t}, X_{t 1}, \ldots, X_{t\left(L^{*} 1\right)}\right)$ where $L^{*}$ was found in the first phase.

From a formal point of view, the proposed ( $\mathrm{L}, \mathrm{p})$ estimation algorithm is described below, step by step:

1: Calculate $(\mathrm{L})=\mathrm{L}+\sum_{\mathrm{i}=0}^{\mathrm{N} 1} \mathrm{X}_{\mathrm{t} i \mathrm{i}} \quad 2 \cdot \sum_{\mathrm{i}=0}^{\mathrm{L} 1} \mathrm{X}_{\mathrm{t} \text { i }}$ for $\mathrm{L} \in\{1,2, \ldots, \mathrm{N}\}$

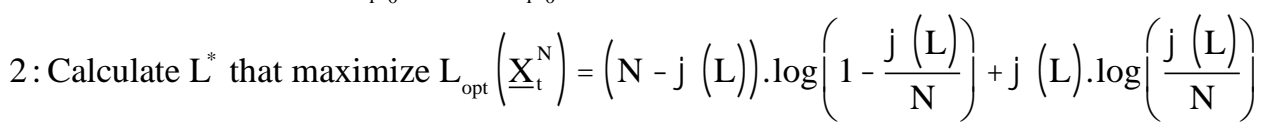

3: If $\mathrm{L}^{*}<\mathrm{L}_{\text {bound }}$ then

4: $\quad \mathrm{p}^{* *}=\frac{1}{\mathrm{~N}} \sum_{\mathrm{i}=0}^{\mathrm{N} 1} \mathrm{X}_{\mathrm{t} \text { i }} \quad \mathrm{L}^{* * *}=\mathrm{L}_{\text {bound }}$

5: Else, for $\mathrm{L} \in\left\{1,2, \ldots, \mathrm{L}^{*}\right\}$,

6: $\quad$ Calculate $\mathrm{L}^{* * *}$ that maximize $\mathrm{L}_{\text {opt }}\left(\underline{\mathrm{X}}_{\mathrm{t}}^{\mathrm{N}}\right)=(\mathrm{N} \quad(\mathrm{L})) \cdot \log \left(1 \frac{(\mathrm{L})}{\mathrm{N}}\right)+(\mathrm{L}) \cdot \log \left(\frac{(\mathrm{L})}{\mathrm{N}}\right)$

7: $\quad$ Let $\mathrm{p}^{* *}=1 \frac{\left(\mathrm{L}^{* *}\right)}{\mathrm{N}}$

$8:$ end if

9: return the pair $\left(\mathrm{L}^{* *}, \mathrm{p}^{* *}\right)$ 
The proposed method for the SU occupancy of the prediction window is simple, and it is based upon the estimation of the pair (L,p), as discussed in previous section. It can be stated as follows: if the estimated $\mathrm{p}$ is less or equal to $1 / 2$, the prediction window is fully used by the $\mathrm{SU}$, since it is more likely that this window is made by 0 's. Otherwise, prediction window is simply not used by SU.

\section{Performance evaluation}

In order to access the quality of the proposed estimation procedure, a computer program was built that:

i. for fixed values for $M$ and $N$, randomly selects several values for $L$ and $p$;

ii. for each one of these pair of values:

a. a long binary sequence with these characteristics is generated;

b. parameters $\mathrm{L}$ and $\mathrm{p}$ are estimated by the proposed method.

iii. these estimated parameters are then confronted with the real ones, and their statistical relative differences are evaluated.

A series of 10000 cases were generated, and for each of them the statistical behavior of relative Lerror, namely $1 \mathrm{~L}_{\text {estimated }} / \mathrm{L}_{\text {real }}$, and P-error, namely, $1 \quad \mathrm{P}_{\text {estimated }} / \mathrm{P}_{\text {real }}$, were evaluated for several values of channel observation errors.

Figure 2 illustrates the complementary cumulative distribution functions of these relative errors for values of observation error ranging from $1 \%$ to $30 \%$. In Figure 2(a) we can see that, if observation error does not exceed 5\%, then the probability the relative L-error does exceed $0 \%$ is $3 \%$, while in Figure 2(b) the probability the relative P-error does exceed $50 \%$ value in $63 \%$ of the cases.

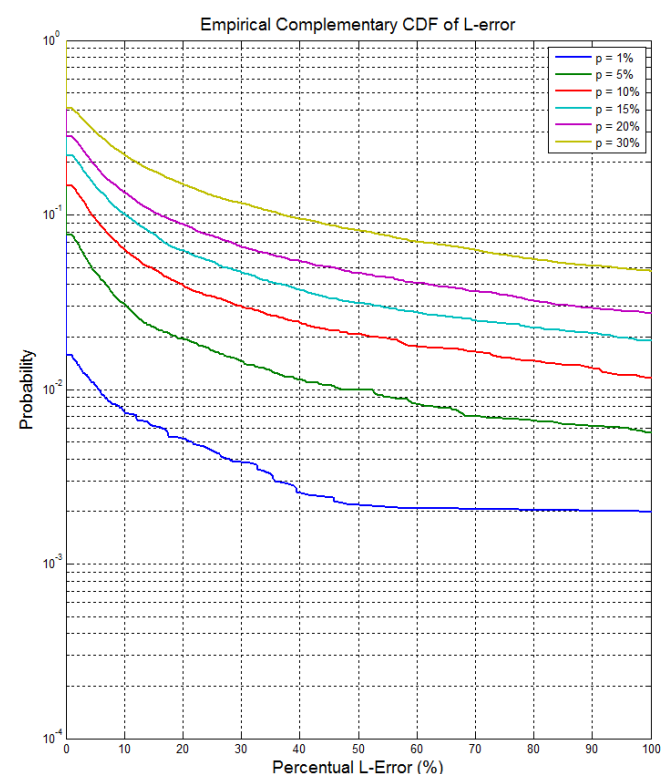

(a)

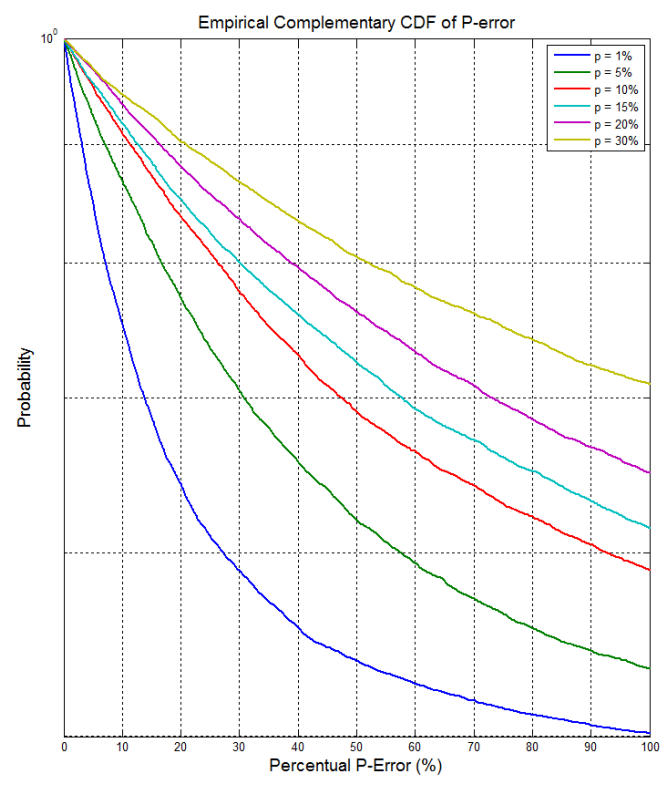

(b) 
Fig. 2. Complementary CDF of L-error and P-error behavior

It is important to notice that the P-error is of little significance in the sense that what matters is if both $\mathrm{P}_{\text {estimated }}$ and $\mathrm{P}_{\text {real }}$ are simultaneously below or above 1/2. According to figure 2(b), if we have measurement errors not superior to $30 \%$, no more than $10 \%$ of the cases violate the mismatch mentioned.

\section{EXPERIMENTAL DATABASE}

In order to produce real data from a typical cognitive radio environment, a measurement campaign was performed in the city of Campinas, São Paulo, Brazil, where the 450-470 MHz frequency band was monitored in the urban environment. Taxi radio companies, highway concessionaires, airlines administrative communications, transport companies and oil refineries use this frequency band for voice services. These are examples of Private Limited Service primary users.

A representative example of the results obtained is shown in Figure 3 for the $457.530 \mathrm{MHz}$ channel that, monitored during the period from 14:19:00 to 15:59:57 hours, had an occupation of 34\% of time. Individual channels percentage of occupancy are displayed on Figure 4. The overall average occupancy during the measurements period was around $26 \%$ of the time, which indicates high idleness in the time domain.

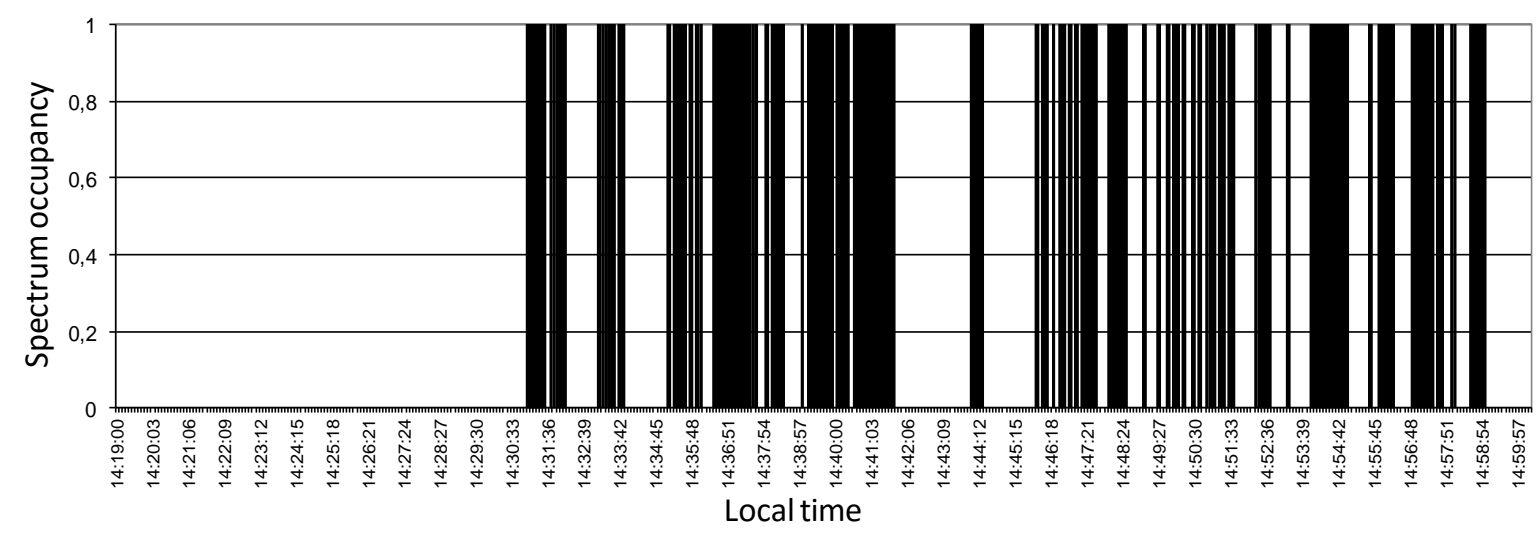

Fig. 3. Time series of spectrum occupancy at $457.530 \mathrm{MHz}$ in Campinas, Brazil 


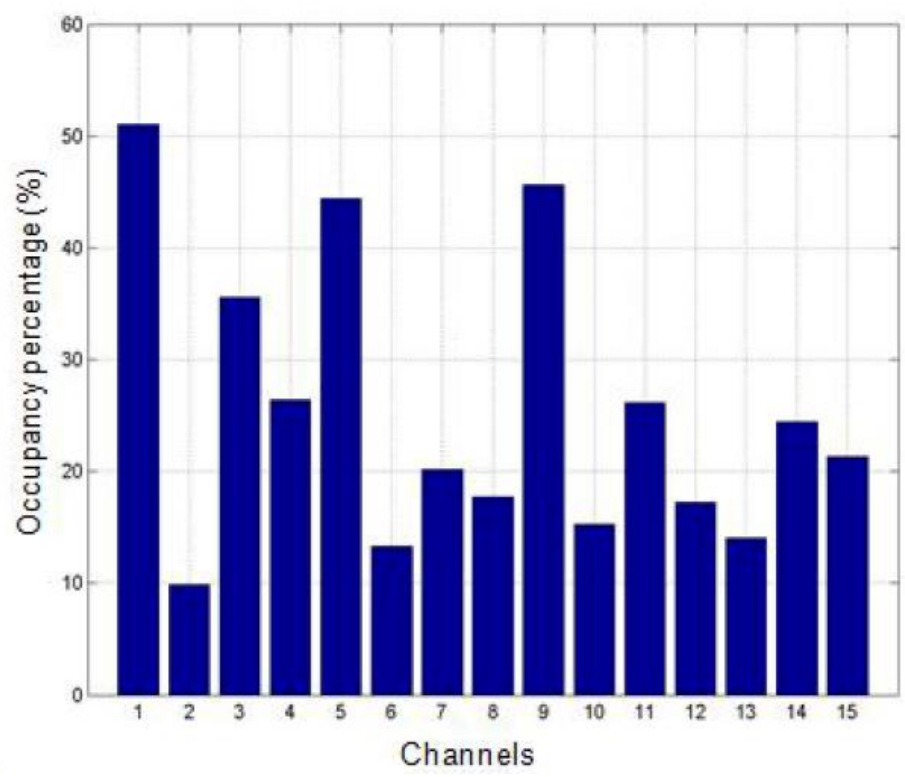

Fig. 4. Channel occupancy in the 450-470 MHz band in Campinas, Brazil

The audio records were individually analyzed to identify the periods of conversation and the black and white spaces within the conversations, as illustrated in Figure 5. Channel occupancy was detected by means of techniques described in [12] and [13] and are also explained in [14]. Although subjected to human interpretation, the eventual errors introduced should not produce significant impact on the results.

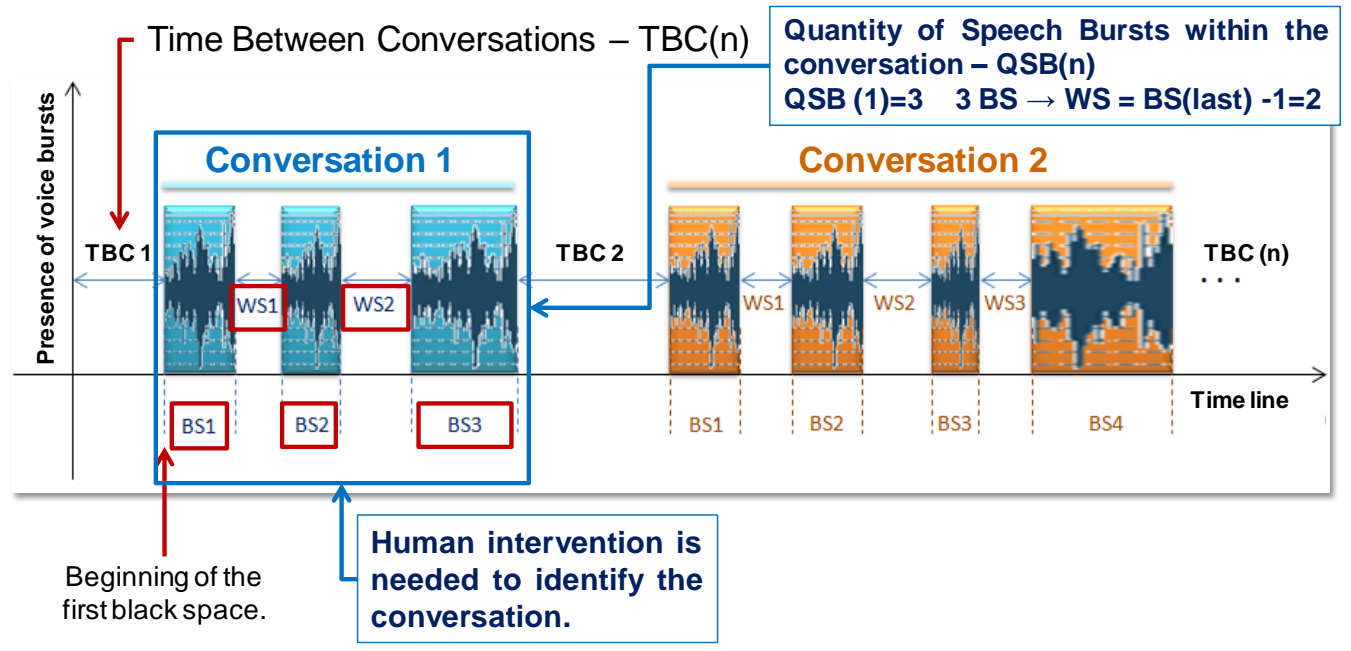

Fig. 5. Definition of the parameters measured

The results of these measurements allowed the statistical assessment of relevant data parameters such as: i) time between conversations, ii) black and white space durations and iii) number of voice bursts in each conversation. Using this characterization, an arbitrary number of random input sequences can be generated for simulation purposes, in which the statistical similarity with the collected real data is totally preserved. It is important to notice that these generated sequences are 
statistically indistinguishable from the original measured sequence thus allowing that collected statistics of performance indicators do present statistical significance.

\section{SiMULATION RESULTS AND COMPARISON WITH OTHER METHODS}

Gulnur et al [6] recently proposed three methods to deal with the spectrum vacancy prediction problem that perform well when compared with other techniques found in the literature. The methods are:

- prediction based on the Pearson correlation coefficient (Method 1);

- linear regression-based prediction (Method 2);

- prediction based on auto-correlation (Method 3).

In this article, they compare the performance of the above methods with those proposed by Zao et al in [8] and simulations results have showed that the former outperforms the latter. Due to this reason, we only compare our proposed method with those in [6].

The performance evaluations discussed here are based on those used in the majority of articles in this field, namely, the System Utility (SU) and the PU Disturbance Ratio (DR) that, for the sake of clearness, are explained below:

- System Utility (SU) is the ratio between the successful use of spectral vacancies and the total white spaces detected. It expresses how efficient the secondary user opportunistically uses the primary user idle periods. Ideally, this value should be as close as possible to $100 \%$.

- PU Disturbance Ratio (DR) is the ratio between the number of slots in which the secondary user has allocated its transmissions by the number of slots that the primary user was transmitting. It expresses the unwanted impact of the secondary user actions on the primary user channel's use. Ideally, this value should be as close as possible to $0 \%$.

Several input sequence of length $10^{7}$ were synthetically generated preserving the statistics observed in the real measured data as above discussed. For each one of them, the mean and standard deviation of the performance indicators DU e DR where evaluated for several values channel status error probability and observation and prediction window sizes. In the majority of cases, the method here proposed outperformed those obtained by the three methods presented in [6].

In order to illustrate a typical comparison result, figures 6 and 7 do present such a comparison for the case where prediction windows respectively have lengths 1000 and 600 and for error probabilities ranging from $0 \%$ to $20 \%$. From there we can observe that the proposed method statistically outperformed all others in both performance indicators.

One interesting by-product of the method here presented and visible in these figures, is its observed insensibility of the System Utility statistics to the chosen range of error probability. For the large number of simulations executed, this stability was observed in most cases. 

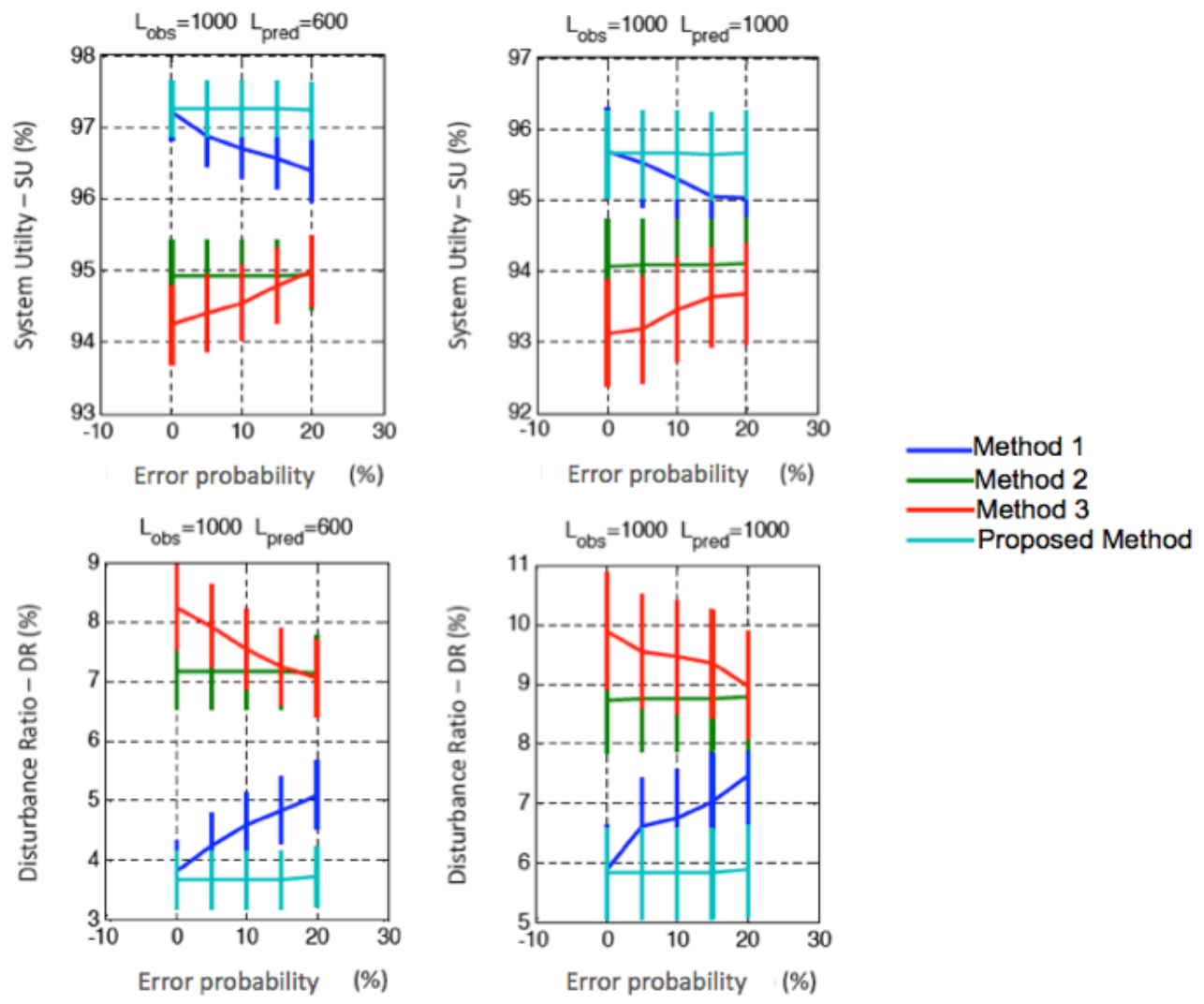

Fig. 6. SU and DU as a function of error probability for observation/prediction window sizes of 1000/600
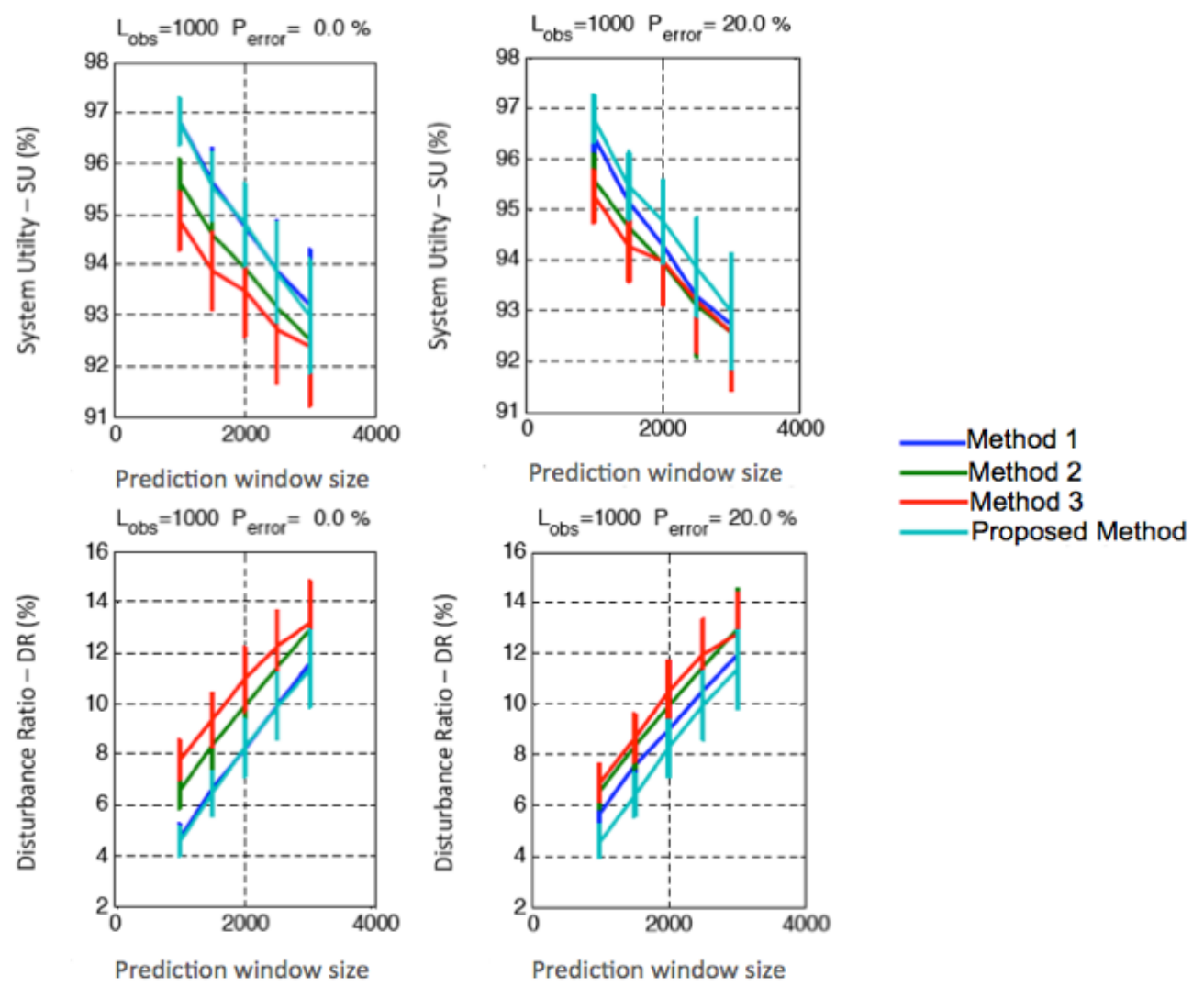

Fig. 7. SU and DU as a function of prediction window size for error probabilities of $0 \%$ to $20 \%$. 


\section{CONCLuSions}

A low computational cost algorithm for spectral vacancies prediction was developed to allow a secondary user (SU) to decide, based on the observation of the primary user (PU) occupancy on a past observation window of fixed size, whether to occupy a future window minimizing the disturbance of the primary user transmissions. The method does not require previous information about PU occupancy statistics, only the data collected in each observation window.

To test the proposed method in a typical scenario, real data collected from a measurement campaign on a voice radio-communications system operating in the 450-470 $\mathrm{MHz}$ band was deployed. Performance tests shown that the method in question outperformed three other methods recently proposed to deal with this problem, as far as System Utility and Disturbance Ratio are the performance indicators.

This comparison has shown the robustness and superiority of the proposed method, for error probabilities in the range $0 \%-20 \%$ and prediction window size from 500 to 3000 samples. As a last detail, it is important to acknowledge that the greater the size of the prediction window, the smaller is the need for probing channel status, which in general requires precious computational resources.

\section{ACKNOWLEDGMENT}

CNPq (National Research Council, Brazil) supported this work under covenant 573939/2008-0 (INCT-CSF).

\section{REFERENCES}

[1] Thomas G., Fast detection of spectral white spaces for cognitive radio networks IEEE MILCOM 2009, Univ. of Louisiana at Lafayette, Lafayette, LA, USA - October, 2009.

[2] Canavitsas A., da Silva Mello L., Grivet M. Spectrum Occupation Modeling on the $450 \mathrm{MHz}$ Band for Cognitive Radios Application. EUCAP 2013 - Gothenburg, Sweden - April, 2013.

[3] Soleimani T., Azad Q., Kahvand M., Sarikhani, R. Handoff reduction based on prediction approach in Cognitive Radio Networks ICCT 2013, - Guilin, China - Nov. 2013

[4] Silverman, S.J. Game Theory and Software Define Radios, Military Communication Conference, 2006 MILCOM 2006, IEEE.

[5] Jing T, Xing X, Cheng W, Huo Y., Cooperative spectrum prediction in multi-PU multi-SU cognitive radio networks. ICCR 2013 - Beijing, China - Nov. 2013

[6] Akhtar, A.N., Rashdi, A., Arif, F., Fusion Based Spectrum Decision Framework for Cognitive Radio Users - Mobile and Multimedia Networks (WoWMoM), 2015 IEEE 16th International Symposium on a World of Wireless - Boston, MA - USA

[7] Barnes S.D., Maharaj M.T., Prediction based channel allocation performance for cognitive radio. International Journal of Electronics and Communications pp.336-345 Volume 68, Issue 4, April 2014.

[8] Zhao Z., Wang L.l, Ding G., Wei S., Enhanced Spectrum Decision Based on the Combination of Sensing and Prediction - Cross Strait Quad-Regional Radio Science and Wireless Technology Conference (CSQRWC), 2011 - Harbin - China

[9] Gulnur S., Uyanik, Canberk B., Oktug S., Predictive Spectrum Decision Mechanisms in Cognitive. Radio Networks GC'12 Workshop: Ad Hoc Networking with MIMO and Cognitive Radio, - Anaheim, California, USA - Dec. 2012.

[10] Gulnur S., Oktug S.A., QoS Based Cooperative Spectrum Utilization in Cognitive Radio Networks, Sarnoff Symposium (SARNOFF), 2012 35th IEEE, Newark, NJ - USA 
[11]Cox D.R., Principles of Statistical Inference, Cambridge University Press, 2006.

[12] Maharjan S., Takada K., Experimental Study of Energy Detector Prototype for Cognitive Radio System. IEICE Technical Report, SR-2007-52(2007-11), Hiroshima International University, Hiroshima, Japan.

[13] Kim J., Jeffrey A., Sensitive White Space Detection with Spectral Covariance Sensing. IEEE Transactions on Wireless Communications, Vol. 9, No. 9, September 2010.

[14]Canavitsas A., da Silva Mello L., Grivet M., White space prediction technique for cognitive radio applications Microwave \& Optoelectronics Conference (IMOC), 2013 SBMO/IEEE MTT-S International, Rio de Janeiro, Brazil. 